\title{
Technetium 99m pyrophosphate radioisotope for diagnosis and prognosis of transthyretin cardiac amyloidosis: A call for collaboration
}

\author{
Adam Castaño, MD, MS, ${ }^{a, b}$ Mathew S. Maurer, MD, ${ }^{b}$ and Sabahat Bokhari, $M^{a}$ \\ a Nuclear Cardiology Laboratory, Center for Advanced Cardiac Care, Division of Cardiology, \\ Columbia University College of Physicians \& Surgeons, New York, NY \\ b Center for Advanced Cardiac Care, Division of Cardiology, Department of Medicine, Columbia \\ University College of Physicians \& Surgeons, New York, NY
}

Received Jan 19, 2017; accepted Jan 20, 2017

doi: 10.1007/s12350-017-0803-5

\section{See related article, pp. 1247-1256}

In this issue of the Journal, Vranian and colleagues ${ }^{1}$ add to the existing literature and reaffirm the association of increased myocardial uptake of phosphate-derived radiopharmaceuticals with poor outcomes among patients with suspected ATTR cardiac amyloidosis. ${ }^{2,3}$ The study confirms the previously reported prognostic value of Tc99m PYP in amyloid cardiomyopathy. ${ }^{3}$ The semiquantitative score of 2 or greater coupled with the absence of an abnormal monoclonal protein (ruling out AL cardiac amyloidosis) was shown to be $100 \%$ specific for diagnosis of ATTR in an international multicenter collaboration of phosphate-derived radiopharmaceutical imaging in cardiac amyloidosis. Thus, technetium-based radionuclide imaging can spare select patients the burden and risk associated with invasive endomyocardial biopsy, which can delay the diagnosis. ${ }^{4}$ The quantitative $\mathrm{H} / \mathrm{CL}$ chest ratio is a measure which has been found to confer $>90 \%$ sensitivity and specificity for detection of ATTR (and differentiation from AL and HFpEF) as well as prognosticate survival in a multicenter analysis of Tc99m PYP cardiac imaging in the United States. ${ }^{3}$

The authors of the current study performed a retrospective analysis in 75 patients with suspected ATTR

Reprint requests: Adam Castaño, MD, MS, Nuclear Cardiology Laboratory, Center for Advanced Cardiac Care, Division of Cardiology, Columbia University College of Physicians \& Surgeons, 622W 168th St. P.H. 10-203, New York, NY, 10032; ac3220@cumc.columbia.edu

J Nucl Cardiol 2018;25:1257-9.

$1071-3581 / \$ 34.00$

Copyright (C) 2017 American Society of Nuclear Cardiology. cardiac amyloidosis who underwent planar Tc99 m PYP cardiac imaging, 48 of whom were reported to have ATTR, 12 with endomyocardial biopsy-proven disease. Myocardial Tc99 m PYP uptake was evaluated on planar images using the established semiquantitative score ${ }^{5}$ and the quantitative $\mathrm{H} / \mathrm{CL}$ chest ratio, ${ }^{6}$ although the authors used a semiquantitative score threshold of $\geq 1$, not $\geq 2$, for detecting ATTR, in part because they used a 3 hour, not 1 hour, imaging time (see below). They found that both the Tc99m PYP semiquantitative score and $\mathrm{H} / \mathrm{CL}$ ratio were associated with mortality with HRs of 1.49 (95\% CI 1.08-2.08, $P=0.016)$ and $5.19(95 \%$ CI 1.46-18.4, $P=0.0111)$, respectively, and with death or heart failure hospitalization with HRs of 1.49 (95\% CI 1.17-1.90) and 3.15 (95\% CI 1.21-8.23), respectively.

However, among the 48 patients reported to have ATTR, the authors showed no association with echocardiographic disease severity or outcomes. These findings contradict prior reports. ${ }^{2,3,7}$ Among 63 patients with hereditary ATTR in Italy, increased myocardial uptake of the Tc99m PYP-like isotope, (99m)Tc-3,3-diphosphono1,2-propanodicarboxylic acid (Tc99m DPD), using a heart-to-whole body ratio, was associated with increased left ventricular wall thickness and lower left ventricular ejection fraction (LVEF), in addition to worse MACEfree survival. ${ }^{2}$ In a study among 121 patients with suspected cardiac amyloidosis in France (55 ATTR and 14 $\mathrm{AL}$ ), increased myocardial retention of another Tc $99 \mathrm{~m}$ PYP-like isotope, (99m)Tc-hydroxymethylene diphosphonate (Tc99m HMDP), using a heart-to-skull retention ratio, was associated with lower LVEF and increased acute heart failure and/or death. ${ }^{7}$ In our own experience among 121 patients with biopsy confirmed ATTR pooled from three centers in the United States, despite a referral population with high pre-test probability of disease and employment of two scan protocols, a Tc99m PYP H/CL 
ratio of 1.6 or greater was associated with lower LVEF, increased interventricular septal wall thickness, lower stroke volume index, lower myocardial contraction fraction, and worse survival. ${ }^{3}$

Potential reasons for the discrepant results in the current study may include its (1) relatively smaller sample size with limited power, (2) inclusion of the different endpoint of heart failure hospitalization compared to previously studied all-cause mortality or MACE endpoints, and (3) differences in the Tc99m PYP scan protocol, which the authors highlight. First, as shown in Table 1, among all 75 patients with suspected ATTR, H/ CL ratio clearly predicted death, and death or heart failure hospitalization. However, when restricting the analysis to the 48 patients with reported ATTR, while the $\mathrm{H} / \mathrm{CL}$ ratio trended in the right direction for prediction of death with a HR of 4.67 , the wide $95 \%$ confidence interval $0.75-29.27$ suggests the study was insufficiently powered to detect a statistically significant signal $(P=0.100)$. A higher event rate in a larger population would have likely yielded a statistically significant signal between increased $\mathrm{H} / \mathrm{CL}$ ratio and death.

Second, H/CL ratio lost predictive ability when heart failure hospitalization or death was employed instead of death alone as an outcome. We commend the authors on exploring heart failure hospitalization as a new outcome among patients with ATTR who underwent Tc99m PYP cardiac imaging. The lack of association may reflect confounding by the complexity of heart failure hospitalization, particularly in this population. Heart failure hospitalizations could have been driven by renal dysfunction, dietary indiscretion, among other insults in addition to myocardial amyloid deposition. It would have been interesting to delineate the precise timing and number of clinical events to account for competing risks between heart failure hospitalization and death, i.e., the number of patients with ATTR who died before heart failure hospitalization could have occurred.
Third, differences in Tc99m PYP scan protocol between the current study and established protocols likely contributed to a lack of signal between increased myocardial uptake and echocardiographic or outcome parameters among patients with ATTR. The current study employed a 3-hour incubation time after patients received an intravenous dose of $20 \mathrm{mCi} \pm 10 \%$ of Tc99m PYP. Other centers have compared a 1- vs 3hour incubation time and demonstrated that the standardized methodology of 1-hour incubation time and only $10 \mathrm{mCi}$ of Tc99m PYP are sufficient for highquality imaging, ${ }^{3,8}$ even in patients with early-stage disease. ${ }^{9}$ The 3-hour incubation time for Tc99 m PYP was derived from original literature of Tc99m PYP for imaging myocardial infarction ${ }^{10,11}$ and from the Tc99m DPD protocol used in Europe, ${ }^{12}$ which calculates a heart-to-whole body ratio. Three hours may not necessarily be the optimal incubation time for the Tc99m PYP $\mathrm{H} / \mathrm{CL}$ ratio, which only requires planar imaging of the chest (not the whole body). In planar chest imaging, a 3hour incubation time is subject to increased background uptake and subsequently lower H/CL chest ratios (mean $\mathrm{H} / \mathrm{CL} 1.58 \pm 0.22$ in the current study) compared to protocols that utilize a shorter 1-hour incubation time (mean H/CL $1.70 \pm 0.44){ }^{3}$ Furthermore, from the perspectives of the patient and healthcare systems efficiency, there is an argument to be made in favor of lower radiation exposure with 10 vs $20 \mathrm{mCi}$ and with a shorter test duration of 1 vs 3 hour.

Now that the phosphate-derived radiopharmaceuticals can noninvasively identify and may prognosticate ATTR cardiac amyloid, it is important to take pause and remember that 'we've only just begun' the work of perfecting our discipline. ${ }^{13,14}$ Given the limitations facing every amyloid specialty center with Tc99m PYP capabilities - relative small study cohorts, late disease presentation, and variation in imaging protocolsmoving forward, it would behoove institutions to collaborate even further by pooling their populations in a priori defined and adequately powered prospective

Table 1. Avenues for partnership in refinement of phosphate-derived radiopharmaceutical for imaging cardiac amyloidosis

1. Standardization of radioisotope dose, incubation time, and analytic ROI methods

2. Determination whether Tc99m PYP has a utility for earlier diagnosis, which may lead to improved outcomes

3. Defining the incremental role of SPECT over planar imaging

4. Understanding differences between the phosphate-derived radiotracers (e.g., Tc99m DPD, Tc99m HMDP, Tc99m PYP)

5. Delineation of the comparative advantages and disadvantages of echocardiographic strain, CMR, and PET in addition to Tc99m PYP in patient-centered management protocols

6. Establishing improved patient outcomes with amyloid-modifying therapies based on cardiac imaging 
studies geared toward our collective management goals. These include raising suspicion of disease in at-risk populations, early and accurate diagnosis, subtyping, prognostication, and tracking response to therapies emerging from clinical trials.

Future avenues of research may include standardization of protocols with respect to radioisotope dose, incubation time, and analytic methods. Additionally, defining the role of Tc99m PYP single-photon emission computed tomography (SPECT) in delineating distribution and quantification of Tc99 $\mathrm{m}$ PYP uptake in the heart will be important as it may add incremental value over current planar Tc99m PYP imaging protocols. Understanding whether there is a relative performance advantage among the different phosphate-derived radiopharmaceuticals (e.g., Tc99m DPD, Tc99m HMDP, Tc99m PYP) for diagnosis, subtyping, and prognostication of ATTR, and whether different protocols are required for optimal imaging will guide optimization of this technique in clinical practice. Harnessing the comparative strengths of other amyloid imaging technologies such as speckle-strain echocardiography, ${ }^{15} \mathrm{CMR},{ }^{16}$ and $\mathrm{PET}^{17}$ will also be necessary for refinement of patient-centered management algorithms. Finally, and perhaps most essential to this work will be the task of improving patient outcomes with amyloid-modifying therapies based on cardiac imaging.

\section{Disclosure}

Dr. Maurer's institution, Columbia University Medical Center, receives funding for research and serving on advisory boards and DSMBs from Pfizer, Inc, Alnylam Pharmaceuticals Inc, ISIS Pharmaceuticals and Prothena Inc.

\section{References}

1. Vranian MN, Sperry BW, Hanna M, et al. Technetium pyrophosphate uptake in transthyretin cardiac amyloidosis: associations with echocardiographic disease severity and outcomes. J Nucl Cardiol 2017.

2. Rapezzi C, Quarta CC, Guidalotti PL, et al. Role of $(99 \mathrm{~m}) \mathrm{Tc}-$ DPD scintigraphy in diagnosis and prognosis of hereditary transthyretin-related cardiac amyloidosis. JACC Cardiovasc Imaging 2011;4:659-70.

3. Castano A, Haq M, Narotsky DL, et al. Multicenter study of planar technetium $99 \mathrm{~m}$ pyrophosphate cardiac imaging: Predicting survival for patients with ATTR cardiac amyloidosis. JAMA Cardiol 2016;1:880-9.

4. Gillmore JD, Maurer MS, Falk RH, et al. Nonbiopsy diagnosis of cardiac transthyretin amyloidosis. Circulation 2016;133:2404-12.

5. Perugini E, Guidalotti PL, Salvi F, et al. Noninvasive etiologic diagnosis of cardiac amyloidosis using 99mTc-3,3-diphosphono1,2-propanodicarboxylic acid scintigraphy. J Am Coll Cardiol 2005;46:1076-84.

6. Bokhari S, Castano A, Pozniakoff T, Deslisle S, Latif F, Maurer MS. (99 m)Tc-pyrophosphate scintigraphy for differentiating light-chain cardiac amyloidosis from the transthyretin-related familial and senile cardiac amyloidoses. Circ Cardiovasc Imaging 2013;6:195-201.

7. Galat A, Rosso J, Guellich A, et al. Usefulness of $(99 \mathrm{~m}) \mathrm{Tc}-$ HMDP scintigraphy for the etiologic diagnosis and prognosis of cardiac amyloidosis. Amyloid 2015;22:210-20.

8. Bokhari S, Morgenstern R, Weinberg R, et al. Standardization of 99mTechnetium pyrophosphate imaging methodology to diagnose TTR cardiac amyloidosis. J Nucl Cardiol 2016.

9. Haq M, Pawar S, Berk JL, Miller EJ, Ruberg FL. Can 99 m-Tcpyrophosphate aid in early detection of cardiac involvement in asymptomatic variant TTR amyloidosis? JACC Cardiovasc Imaging 2016.

10. Corbett JR, Lewis SE, Wolfe CL, et al. Measurement of myocardial infarct size by technetium pyrophosphate single-photon tomography. Am J Cardiol 1984;54:1231-6.

11. Rude RE, Parkey RW, Bonte FJ, et al. Clinical implications of the technetium-99m stannous pyrophosphate myocardial scintigraphic "doughnut" pattern in patients with acute myocardial infarcts. Circulation 1979;59:721-30.

12. Rapezzi C, Quarta CC, Guidalotti PL, et al. Usefulness and limitations of 99mTc-3,3-diphosphono-1,2-propanodicarboxylic acid scintigraphy in the aetiological diagnosis of amyloidotic cardiomyopathy. Eur J Nucl Med Mol Imaging 2011;38:470-8.

13. Bhambhvani P, Hage FG. Nuclear imaging of cardiac amyloidosis. 'We've only just begun'. J Nucl Cardiol 2016.

14. Gibbons RJ. What is the evidence? A call for scientific rigor. J Nucl Cardiol 2016.

15. Phelan D, Collier P, Thavendiranathan $P$, et al. Relative apical sparing of longitudinal strain using two-dimensional speckletracking echocardiography is both sensitive and specific for the diagnosis of cardiac amyloidosis. Heart 2012;98:1442-8.

16. Fontana M. Prognosis in cardiac amyloidosis by LGE: Ready for prime time? JACC Cardiovasc Imaging 2016;9:687-9.

17. Pilebro B, Arvidsson S, Lindqvist $\mathrm{P}$, et al. Positron emission tomography (PET) utilizing Pittsburgh compound B (PIB) for detection of amyloid heart deposits in hereditary transthyretin amyloidosis (ATTR). J Nucl Cardiol 2016. 